\title{
Quantum Surface Control of Trapped Bose-Einstein-Condensates
}

\author{
Quan-Fang Wang \\ Mechanical and Automation Engineering, The Chinese University of Hong Kong, Shatin, \\ N.T., Hong Kong \\ E-mail: quanfangwang@hotmail.com
}

\begin{abstract}
In this work, quantum control of trapped Bose-Einstein-Condensates (BEC) is considered at matter surface. For particles at BEC status, quantum system is described by Gross-Pitaevskii equation, experimental control of BEC is happened at physics field, and achieved at laboratory. At theoretic aspect, control of trapped condensates is not sufficiently investigated at academic level. What we interest is applying control theory to BEC trapped on the surface (metallic, crystal).

At optical lattice, particles are trapping by constrained forces at cooling technique, and temporally take the same quantum states, such kind of condensates phenomena had already been surveyed at a variety of areas. The most works are reported on free BEC particles, quite natural question is arising on the surface science: BEC particles created,detected, and placed on a certain chemical surface, control of trapped particles is difference or not? We are curious about optical and mechanical constraints take action together on particles.

In the viewpoint of quantum control realm, our purpose is to apply optimal control theory (OCT) to trapped Bose-Einstein-Condensates as they are occurred at surface. In the framework of variational theory at complex Hilbert spaces, prove the existence of quantum optimal control, and characterize optimal control using optimality (Euler-Lagrange) system. Control variables for trapped BEC contain three functions: one is electro-magnetic force; another is external constraint from optical equipment (optical frequency, lattice number); third is quantum mechanics against gravitational force, which making BEC particles stay at surface stationary. Review the literatures, electro-magnetic-optical controls are extremely considered at last couple of years. Gravitational control is rarely considered.

Further extension of the work is to do real-time computer-aided BEC control at matter surface. Computational approach for simulation of BEC control at two and three dimensions would be a promise direction.
\end{abstract}

PACS numbers: 67.85.Jk, 03.65.Ta, 04.60.-m, 79.20.Rf

Keywords: Quantum control, Bose-Einstein-Condensates, Gross-Pitaevskii equation, Surface control.

Submitted to: J. Phys. A: Math. Theor. 


\section{Physical Background}

1990, the bosonic atoms can be reached Bose-Einstein-Condensates (BEC) in lab experiments for various particles (e.g. ${ }^{87} \mathrm{Rb},{ }^{23} \mathrm{Na}$ ). After that, the researches of BEC had been considered at the field of quantum physics and relative areas for a long time [1],[2],[3],[4]. Free trapped BEC had well been investigated at the optical lattices [5]. In fact, at the physical lab, if an ultra-cold vapor of bosonic atoms are trapped in magnetic well, pure condensates will be created as they are cooled to a temperature below the BEC threshold. After that creation, these BEC are located into a optical lattice potential which can be done at current laboratory experimental condition. In general, control of Bose-Einstein-Condensate has not been sufficiently taken account into research topic not only at the field of system control, but also at the particle physics and quantum physics .

To do control of BEC, first need to be considered the purpose of control, that is means what we want the BEC do for us? if just to create a BEC at optical lattices, no problem at all now for a cold-atom system confined in a magneto-optical trap. Then, what the BEC can do for much more usefulness? Theoretical as well as computational free control of BEC had been reported in our former works [6] and [7]. In this article, we take the BEC as they are occurred at a matter surface (metal, crystal, etc). Whether these kind of control is different than free control case. It is one of the aim to find the meaningful of control of BEC, and make sense of BEC at realistic world.

Physics experiments for creating atomic BEC at surface: such as ${ }^{23} \mathrm{Na}$ at Si surface; ${ }^{87} \mathrm{Rb}$ at $\mathrm{Si}$; Ti; Au surface; ${ }^{23} \mathrm{Na}$ at ${ }^{4} \mathrm{He} ;{ }^{3} \mathrm{He}$ surface; ${ }^{133} \mathrm{Cs}$ at $\mathrm{Si}$ surface, and so on.

In lab, temperature of creating Bose-Einstein-Condensate $T_{b}=\frac{h^{2}}{2 \pi m k}\left(\frac{N}{2.612 V}\right)^{2 / 3}$; the number of atoms in a BEC $N(T)=N\left\{1-\left(T / T_{b}\right)^{3 / 2}\right\}$; average de-Broglie wave length $\lambda=\frac{h}{\sqrt{3 m k T}}$.

\section{Trapped Bose-Einstein-Condensates (BEC)}

At first, we show the model of trapped Bose-Einstein-Condensates citing [8]. Suppose $N$ number particles are trapped in a condensates, each particle as $P_{n}$ for $n=1,2, \cdots, N$. BoseEinstein-Condensates is a unusual state of matter in which bosons collectively occupy the energetic ground state of a quantum system. In physics field, BEC at matter surface had been created by atom chip, such as silicon wafer that has wires deposited on its surface. By passing a current through these wires, a magnetic field is produced which is used to create and manipulate Bose-Einstein-Condensates. To trap a free BEC in science vacuum chamber (cf. Fig.1), hit atoms with surface rather than hitting the surface with atom. 

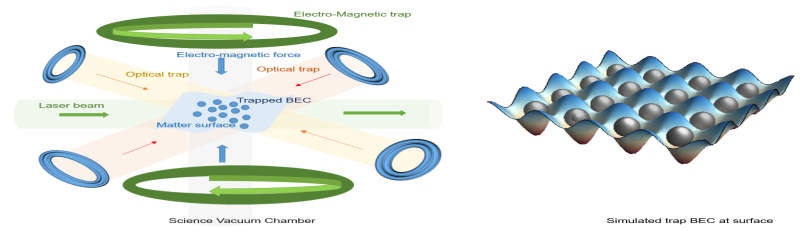

Fig. 1. Science Vacuum Chamber, Simulation of Trapped BEC

Suppose there is no chemical reaction taking place between the particles and matter surface, then, without lost of generality, Bose-Einstein-Condensates can be taken as Gross-Pitaevskii (G-P) equation in the form of a nonlinear Schrödinger equation (cf. [3], [4]) for $\mathbf{x}=\left(x_{1}, x_{2}\right) \in$ $\mathbf{R}^{2}$,

$$
\mathbf{i} \hbar \frac{\partial \psi_{n}}{\partial t}=-\frac{\hbar^{2}}{2 m} \Delta \psi_{n}+\left(V_{n}^{1}(\mathbf{x})+V_{n}^{2}(\mathbf{x})+V_{n}^{3}(\mathbf{x})\right) \psi_{n}+N v\left|\psi_{n}\right|^{2} \psi_{n},
$$

for $n=1,2, \cdots, N$. $\hbar$ is Planck constant, $m$ is atomic mass, $\psi_{n}(t, \mathbf{x})$ is condensate wave function of $n$-th particle $P_{n}$ at BEC, $N$ is total number of condensed atoms, and $v=4 \pi \hbar^{2} a_{s} / m$ is interacting constant of ground state atoms, where $a_{s} \in \mathbf{R}$ is $s$ wave characteristic scattering length (e.g. $a_{s}=2.75,5.77,-1.45 \mathrm{~nm}$ for ${ }^{23} \mathrm{Na},{ }^{87} \mathrm{Rb},{ }^{7} \mathrm{Li}$ ). Notice that, in mean-field theory, one can substitute $\psi_{n}=\exp (-\mathbf{i} \mu t / \hbar) \psi_{n}(\mathbf{x})$ in system (1) to get time-independent G-P equation having $-\mu \psi_{n}$ term of chemical potential $\mu$.

A lot of physical control at lab by adjustment of the parameter of scattering length $a_{s}$ incident. For quantum control purpose, total external force is unified and set $V_{n}(t, \mathbf{x})=$ $V_{n}^{1}(t, \mathbf{x})+V_{n}^{2}(t, \mathbf{x})+V_{n}^{3}(t, \mathbf{x})$.

1). The external potential $V_{n}^{1}(\mathbf{x})$ is electro-magnetic field for creating BEC of $\mathrm{N}$ particle $\left(P_{1}, \cdots, P_{N}\right)$, in general, $V_{n}^{1}(\mathbf{x})=m \sigma_{0}^{2} \frac{|\mathbf{x}|^{2}}{2}$, trapping frequency $\sigma_{0}=2 \pi\left|\mathbf{x}_{0}\right|^{2} \in \mathbf{R}^{2}$. A harmonic oscillator can be configured as $V_{n}^{1}=\frac{m}{2}\left(\omega_{x_{1}}^{2} x_{1}^{2}+\omega_{x_{2}}^{2} x_{2}^{2}\right)$.

2). In lab, optical potential [9] for particle $P_{n}$ (cf. [5]) can be configured as $V_{n}^{2}(\mathbf{x})=$ $\mu \sum_{k=1}^{2} \frac{\hbar^{2} \mathbf{x}^{2}}{m} \sin ^{2}\left(x_{k} x_{k}\right), x_{k} \in \mathbf{R}^{2}$, and $\mu>0$ is a dimensionless parameter for representing the depth of optical lattice. The trapped field can adjust the optical frequencies along the directions of $x_{1}, x_{2}$ axis. Other kind of optical apparatus can be also confining, e.g. $V_{n}^{2}=\hbar \frac{\Omega(t)}{2 \Delta}\left(1+\cos \left(2 k_{L} \mathbf{x}-\delta_{L} t\right)\right): k_{L}$ wave vector; $\delta_{L}$ difference of two beam; $\Omega_{0}$ single photon Rabi frequency; $\Delta$ detuning.

3 ). The mechanical gravitational force $V_{n}^{3}(\mathbf{x})$ is making these particles staying (in vertical and horizontal directions) at a matter surface, which indicated the gravity in gravito-magnetic trap or gravito-optical trap. The magnetic-optical trap known as optical molasses.

Here, we need to discuss atom surface interaction. Such as, intuitive and no consideration of surface structure, $r$ is separation or displacement from the atom to center of trap, then for 
local speed of sound $c$ (e.g. ${ }^{23} \mathrm{Na}$ with density $2 \times 10^{12} \mathrm{~cm}^{-3}, c \sim 0.6 \mathrm{~mm} / \mathrm{s}$ ) and frequency $\omega_{0}$, atom surface potential can be written

a). $r \geqslant c / \omega_{0}: V_{\text {Casimir Polder }}=-C_{4}(\rho) / r^{4}$, and $C_{4}(\rho)$ mediated by structure of surface, e.g. $C_{4}^{S i}=6.2 \times 10^{-56} \mathrm{Jm}^{4}$ for bulk silicon.

b). $r \geqslant c / \omega_{0}$ and $r>\lambda T: V_{\text {Lifshitz }}=-C_{L}(\rho) T / r^{3}$.

c). $r \leqslant c / \omega_{0}: V_{\text {van der Waals London }}=-C_{3}(\rho) / r^{3}$, e.g. $C_{3}=6.9 \times 10^{-48}$.

For ${ }^{87} \mathrm{Rb}, V_{C P}=1 / r^{4}, C_{v d w}=1 / r^{3}$. Atom surface interaction (many-body interaction) could be atom-atom interaction if the surface broken down to its constituent atom.

The disadvantage of gravity in BEC creation, destructure and reduce the gravitational force, the influence knows as "gravitational sag". The confined gravito-magnetic trap is to against the gravity to create a BEC in Vacuum Chamber. At solid surface, such as silicon, atom collide to surface at low or zero velocity, and near the surface, non-condensed fraction of atomic cloud is reduced by the "surface evaporation" effect, in which adsorption preferentially remove the hottest atom from the cloud.

Resultantly, BEC system (1) is representing an balance status of interacting $N$ particles BEC condensate trapped by cooling technique: optical lattice; electro-magnetic force; and gravito-magnetic force (atom surface interaction). Our mission is to discuss three difference control inputs to the complex G-P equation (1) which is formulated of BEC condensate at matter surface.

\section{Interaction effects (pseudopotential)}

$1 D$ Two particles overlap interaction, an effective 1D contact potential $V_{\text {int }}\left(r_{n}, r_{n^{\prime}}\right)=$ $g_{1 D} \delta\left(r_{n}-r_{n}^{\prime}\right), r_{n}, r_{n}^{\prime}$ is the distance from the atom to trap center, and $g_{1 D}$ is an effective $1 \mathrm{D}$ coupling strength $g_{1 D}=2 a_{s} h \sqrt{v v^{\prime}}, a_{s}$ is scattered length $h$ is Planck constant. $v, v^{\prime}$ are trapped frequencies at $r_{n}, r_{n}^{\prime}$

2D All the particles get forces to support their stay at surface, to each particle $P_{n}$ located at the distance of $r_{n}$. The effective interaction between the bosons, is obtained in the long wavelength approximation to be

$$
V_{n}^{3}\left(r_{n}-r_{n}^{\prime}\right)=\frac{4 \pi \hbar^{2} a_{s}}{m} \delta\left(r_{n}-r_{n}^{\prime}\right)
$$

where $\hbar$ is Planck's constant, $a_{s}$ is the s-wave scattered length.

3D Notice that, free particles in spatial 3D, $g_{3 D}=\frac{4 \pi}{\omega x_{2} x_{3}} g_{1 D}$, then (2) valid.

Interaction effects is taking the similar coefficient as the last term of (1) which is the interaction of two bosons of particles $i, j \in\{1,2, \cdots, N\}$.

$\mu$ Chemical Potential: usual $\mu=\Omega \cdot L$ for vortex trap; $\mu=m \sum_{k=1}^{3} \omega_{k}^{2} x_{k}^{2} / 2$ for spherical trap; many-body $\mu=\delta E / \delta N$ to make total number of condensed particles $N=\int\left|{\underset{n=1}{\pi}}_{n}^{N}\right|^{2} d V$, $E$ is energy function. Two dimensional rotating $\mu=1-\Omega^{2}$. For uniform $\mu=N v$. $\mu$ can 
be considered either a Lagrange multiplier or a parameter in the zero temperature groundcanonical thermodynamics potential.

Hamiltonian. Get notation complex scalar function $\psi_{n}(t, \mathbf{x})$ and operator $\hat{\psi}_{n}(t, \mathbf{x})$, then $\psi_{n}(t, \mathbf{x})=\left\langle\hat{\psi}_{n}(t, \mathbf{x})\right\rangle$. The Hamiltonian $\mathbf{H}_{n}$ for each particle $P_{n}$ at a BEC, then many-body system Hamiltonian

$$
\begin{aligned}
\mathbf{H}=\sum_{n=1}^{N} \mathbf{H}_{n}= & \sum_{n=1}^{N}\left\{\int \hat{\psi}_{n}^{*}(\mathbf{x})\left[-\frac{\hbar^{2}}{2 m} \Delta+V_{n}(\mathbf{x})\right] \hat{\psi}_{n}(\mathbf{x}) d \mathbf{x}\right. \\
& \left.+N \frac{4 \pi \hbar^{2} a_{s}}{2 m} \iint \hat{\psi}_{n}^{*}(\mathbf{x}) \hat{\psi}_{n}^{*}(\mathbf{y}) \hat{\psi}_{n}(\mathbf{x}) \hat{\psi}_{n}(\mathbf{y}) d \mathbf{x} d \mathbf{y}\right\} .
\end{aligned}
$$

$\hat{\psi}_{n}^{*}$ is conjecture (field operator) of $\hat{\psi}_{n}$, and $\hat{\psi}_{n}$ or $\hat{\psi}_{n}{ }^{*}$ is annihilate or create particle. $\left[\hat{\psi}(\mathbf{x}), \hat{\psi}^{*}(\mathbf{y})\right]=\delta(\mathbf{x}-\mathbf{y}),\left[\hat{\psi}^{*}(\mathbf{x}), \hat{\psi}^{*}(\mathbf{y})\right]=0$.

Lagrangian density function. Total Lagrangian function for many-body $N$ particles in a BEC

$$
\begin{aligned}
\mathscr{L}= & \sum_{n=1}^{N} \mathscr{L}\left(\psi_{n}(t, \mathbf{x})\right)=\sum_{n=1}^{N}\left\{\frac{1}{2} \mathbf{i} \hbar\left[\frac{\partial \psi_{n}^{*}(t, \mathbf{x})}{\partial t} \psi_{n}(t, \mathbf{x})-\psi_{n}^{*}(t, \mathbf{x}) \frac{\partial \psi_{n}(t, \mathbf{x})}{\partial t}\right]\right. \\
& \left.+\frac{\hbar^{2}}{2 m}\left|\nabla \psi_{n}(t, \mathbf{x})\right|^{2}+V_{n}(t, \mathbf{x})\left|\psi_{n}(t, \mathbf{x})\right|^{2}+\frac{1}{2} N v\left|\psi_{n}(t, \mathbf{x})\right|^{4}\right\} .
\end{aligned}
$$

\section{Mathematical Setting}

Second, we give mathematical setting at the framework of variational method in complex Hilbert spaces citing [10]-[12]. Let $\Omega$ be an open bounded set of $\mathbf{R}^{2}$ and $Q=(0, T) \times \Omega$ for $T>0,(t, \mathbf{x}) \in Q$. In G-P system (1), $V_{n}(t, \mathbf{x})$ is total control input acting at particle $P_{n}$, and control $V_{n}(t, \mathbf{x})$ is depended on spatial variable $\mathbf{x}$ and time $t$. For complex-valued function $\psi_{n}$, define complex solution space $\mathbb{L}^{2}(\Omega)$ and $\mathbb{H}_{0}^{1}(\Omega)$ corresponding to $L^{2}(\Omega)$ and $H_{0}^{1}(\Omega)$, use the notations $\mathbb{H}=\mathbb{L}^{2}(\Omega), \mathbb{V}=\mathbb{H}_{0}^{1}(\Omega)$ for two complex Hilbert spaces. Further, $\mathbb{V}^{\prime}$ is complex conjugate space of $\mathbb{V}$. Actually, $(\mathbb{V}, \mathbb{H})$ is a complex Gelfand triple spaces $\mathbb{V} \hookrightarrow \mathbb{H} \hookrightarrow \mathbb{V}^{\prime}$, two embeddings are continuous, dense and compact. For $\psi_{n}=\psi_{n}^{1}+\mathbf{i} \psi_{n}^{2} \in \mathbb{L}^{2}(\Omega), \psi_{n}^{1}, \psi_{n}^{2} \in$ $L^{2}(\Omega)$, define norm of $\psi_{n}$ in complex space $\mathbb{L}^{2}(\Omega)$ as

$$
\left\|\psi_{n}\right\|_{\mathbb{L}^{2}(\Omega)}=\left(\left\|\psi_{n}^{1}\right\|_{L^{2}(\Omega)}^{2}+\left\|\psi_{n}^{2}\right\|_{L^{2}(\Omega)}^{2}\right)^{\frac{1}{2}} .
$$

If $\psi_{n}=\psi_{n}^{1}+\mathbf{i} \psi_{n}^{2} \in \mathbb{L}^{2}(\Omega)$ and $\phi_{n}=\phi_{n}^{1}+\mathbf{i} \phi_{n}^{2} \in \mathbb{L}^{2}(\Omega)$, then inner product of $\psi_{n}$ and $\phi_{n}$ in complex space $\mathbb{L}^{2}(\Omega)$ can be defined by

$$
\left.\left(\psi_{n}, \phi_{n}\right)_{\mathbb{L}^{2}(\Omega)}=\left(\left(\psi_{n}^{1}, \phi_{n}^{1}\right)_{L^{2}(\Omega)}+\left(\psi_{n}^{2}, \phi_{n}^{2}\right)\right)_{L^{2}(\Omega)}\right)+\mathbf{i}\left(\left(\psi_{n}^{2}, \phi_{n}^{1}\right)_{L^{2}(\Omega)}-\left(\psi_{n}^{1}, \phi_{n}^{2}\right)_{L^{2}(\Omega)}\right) .
$$

For $\psi_{n} \in \mathbb{H}_{0}^{1}(\Omega)$, the norm of $\psi_{n}$ in complex space $\mathbb{H}_{0}^{1}(\Omega)$ is

$$
\left\|\psi_{n}\right\|_{\mathbb{H}_{0}^{1}(\Omega)}=\left(\left\|\psi_{n}^{1}\right\|_{H_{0}^{1}(\Omega)}^{2}+\left\|\psi_{n}^{2}\right\|_{H_{0}^{1}(\Omega)}^{2}\right)^{\frac{1}{2}} .
$$

If $\psi_{n}, \phi_{n} \in \mathbb{H}_{0}^{1}(\Omega)$, then inner product of $\psi_{n}$ and $\phi_{n}$ in complex space $\mathbb{H}_{0}^{1}(\Omega)$ is defined as

$$
\left\langle\psi_{n}, \phi_{n}\right\rangle_{\mathbb{H}_{0}^{1}(\Omega)}=\left\langle\psi_{n}^{1}, \phi_{n}^{1}\right\rangle_{H_{0}^{1}(\Omega)}+\left\langle\psi_{n}^{2}, \phi_{n}^{2}\right\rangle_{H_{0}^{1}(\Omega)}
$$


Set ground state of $n$-th particle $P_{n}$ as $\psi_{n}\left(0, V_{n}\right)=\psi_{n 0}$. The total ground state $\psi(0, \mathbf{V})=\psi_{0}$, and $\psi(\mathbf{V})=\left(\psi_{1}, \psi_{2}, \cdots, \psi_{N}\right)$. Denote $\mathscr{H}=\mathbb{H}^{N}=\mathbb{L}^{2}(\Omega)^{N}$, and $\mathscr{V}=\mathbb{V}^{N}=\mathbb{H}_{0}^{1}(\Omega)^{N}$.

Definition 1 [Solution space] Complex space $W(0, T)$ is said as solution space of $\psi$

$$
W(0, T)=\left\{\psi \mid \psi \in L^{2}(0, T ; \mathscr{V}), \psi^{\prime} \in L^{2}\left(0, T ; \mathscr{V}^{\prime}\right)\right\} .
$$

The norm of $\psi_{n}$ in complex space $W(0, T)$ can be defined as

$$
\|\psi\|_{W(0, T)}=\left(\|\psi\|_{L^{2}(0, T ; \mathscr{V})}^{2}+\left\|\psi^{\prime}\right\|_{L^{2}\left(0, T ; \mathscr{V}^{\prime}\right)}^{2}\right)^{\frac{1}{2}} .
$$

If $\psi, \phi \in W(0, T)$, then definition of inner product is

$$
\langle\psi, \phi\rangle_{W(0, T)}=\langle\psi, \phi\rangle_{\mathscr{V}}+\left\langle\psi^{\prime}, \phi^{\prime}\right\rangle_{\mathscr{V}^{\prime}}
$$

Thus, $W(0, T)$ is a complex Hilbert space equipped by above norm and inner product.

Definition 2 [Weak solution] A function $\psi$ is said as weak solution of BEC system in the form of G-P equation (1) for $N$ particles $\left(P_{1}, \cdots, P_{N}\right)$, if $\psi \in W(0, T)$ and satisfy

$$
\int_{0}^{T} \int \mathbf{i} \hbar \frac{\partial \psi}{\partial t} \eta d \mathbf{x} d t=\frac{\hbar^{2}}{2 m} \int_{0}^{T} \int \nabla \psi \nabla \eta \mathbf{x} d t+\int_{0}^{T} \int \mathbf{V} \psi \eta d \mathbf{x} d t+N v \int_{0}^{T} \psi^{2} \psi \eta d \mathbf{x} d t
$$

where $\eta$ is a arbitrary test function by the means of distribution in space $\mathscr{D}^{\prime}(0, T)^{N}, \eta \in$ $C^{1}(0, T ; \mathscr{V})$ and $\eta(T)=0$. The formulation (3) is said weak form of BEC system (1).

The existence theorem of weak solution.

Theorem 3 [Existence theorem of weak solution] For $\psi_{0} \in \mathscr{V}$ of $N$ particle $\left(P_{1}, \cdots, P_{N}\right)$, then there exists a unique weak solution $\psi \in W(0, T)$ for G-P equation (1).

Our trapping manipulation $\left(V_{n}^{1}, V_{n}^{2}, V_{n}^{3}\right)$ is worked and valid for finding a wave function solution of G-P equation.

\section{Quantum Surface Control of BEC}

Third, let's to apply quantum control theory to BEC and give the proof. Suppose $Q=$ $[0, T] \times \Omega$ and set $V_{n}=V_{n}^{1}+V_{n}^{2}+V_{n}^{3}, \mathscr{U}=L^{2}(Q)$ is the space of controls $V_{n}$, and $\mathscr{U}_{a d}$ is a admissible set of $\mathscr{U}$. Set control $\mathbf{V}=\left(V_{1}, \cdots, V_{n}, \cdots, V_{N}\right)$, and corresponding optimal control $\mathbf{V}^{*}=\left(V_{1}^{*}, \cdots, V_{n}^{*}, \cdots, V_{N}^{*}\right)$. Denote $\mathbf{U}=\mathscr{U}^{N}$ and $\mathbf{U}_{a d}=\mathscr{U}_{a d}^{N}$, then $\mathbf{V} \in \mathbf{U}$. The particles wave motion make us to do terminal observation by photograph, scanning electron micrograph, or computer monitor at final time $T, t_{f}$. The cost function of $n$-th particle for G-P system (1) can be composed in the form of

$$
J\left(V_{n}\right)=\varepsilon^{1}\left\|\psi_{n}^{f}\left(V_{n}\right)-\psi_{n}^{\mathrm{target}}\left(V_{n}\right)\right\|_{\mathbb{H}}^{2}+\varepsilon^{2}\left\|V_{n}\right\|_{\mathscr{U}}^{2} .
$$

In criteria function (4), $V_{n} \in \mathscr{U}_{a d}, \psi_{n}^{\text {target }}\left(V_{n}\right)$ is target state, $\psi_{n}^{f}\left(V_{n}\right)$ is observed final state of particle $P_{n}$ at final time $t_{f} \cdot \varepsilon^{i}, i=1,2$ are weighted coefficients for balancing the values of system inherent cost and control running cost. In summary, the total cost function $J(\mathbf{V})=\sum_{n=1}^{N} J\left(V_{n}\right)$ for a condensate of $N$ particles can be represented as

$$
J(\mathbf{V})=\varepsilon^{1}\left\|\psi^{f}(\mathbf{V})-\psi^{\text {target }}(\mathbf{V})\right\|_{\mathscr{H}}^{2}+\varepsilon^{2}\|\mathbf{V}\|_{\mathbf{U}}^{2}
$$


In criteria function (5), $\mathbf{V} \in \mathbf{U}_{a d}, \psi^{\text {target }}(\mathbf{V})=\left(\psi_{1}^{\text {target }}\left(V_{1}\right), \cdots, \psi_{N}^{\text {target }}\left(V_{N}\right)\right)$ is target state, $\psi^{f}(\mathbf{V})=\left(\psi_{1}^{f}\left(V_{1}\right), \cdots, \psi_{N}^{f}\left(V_{N}\right)\right)$ is observed final state of $N$ particle at final time $t_{f}$. Hence, we have $3 N$ controls term of $N$ trapped particles.

Two fundamental problems of quantum optimal control for Bose-Einstein-Condensates at surface:

i). Find quantum optimal control $\mathbf{V}^{*}$ for G-P system (1).

ii). Find Eular-Langrange system for $\mathbf{V}^{*}$.

For $N$ particle $\left(P_{1}, \cdots, P_{N}\right), \mathbf{V}^{*}$ is said as quantum optimal control for G-P system (1) to total cost function (5).

Theorem 4 [Existence of quantum optimal control] For $\psi_{0} \in \mathscr{V}$ of $N$ particles $\left(P_{1}, \cdots, P_{N}\right)$, if $\mathbf{U}_{a d}$ is closed convex (bounded) admissible subset of $\mathbf{U}$, then there exists at least one quantum optimal control $\mathbf{V}^{*}$ of Bose-Einstein-Condensates system (1) subject to cost function (5).

Note that, all the trapping methodologies $\mathbf{V}$ are worked at low temperature $(\sim n K)$ to control atoms, from create a BEC at surface; get enough time to observe low or zero velocity of atoms (density of atom $\uparrow$, velocity of atom $\downarrow$ ); to release atoms from a BEC status.

Proof. Set $J=\inf _{\mathbf{V} \in \mathbf{U}_{a d}} J(\mathbf{V})$, since $\mathbf{U}_{a d}$ is non-empty, there is a sequence $\left\{\mathbf{V}^{k}\right\}$ in $\mathscr{U}_{a d}$ such that $\inf _{\mathbf{V} \in \mathbf{U}_{a d}}, J(\mathbf{V})=\lim _{k \rightarrow \infty} J\left(\mathbf{V}^{k}\right)=J$. Since $\{J(\mathbf{V})\}$ is bounded in $\mathbf{R}^{+}$, and $\mathbf{U}_{a d}$ is closed and convex (bounded) subset of $\mathbf{U}$, there exist a subsequences $\left\{\mathbf{V}^{k^{\prime}}\right\}$ of $\left\{\mathbf{V}^{k}\right\}$ can be extracted, and exist a $\mathbf{V}^{*} \in \mathbf{U}_{a d}$, such that

$$
\mathbf{V}^{k^{\prime}} \rightarrow \mathbf{V}^{*} \text { weakly in } \mathbf{U} \text { as } k^{\prime} \rightarrow \infty .
$$

From the existence Theorem 3 of weak solution to get estimate $\|\psi\|_{\mathscr{H}}^{2}+\|\psi\|_{\mathscr{V}}^{2}$ is bounded for $\psi$ of $N$ particles $\left(P_{1}, \cdots, P_{N}\right)$. For control $\mathbf{V}^{k^{\prime}}$, from boundedness of $\mathbf{U}_{a d}$ that

$$
\psi\left(\mathbf{V}^{k^{\prime}}\right) \text { is bounded in } L^{2}(0, T ; \mathscr{H}) \cap L^{2}(0, T ; \mathscr{V}) .
$$

Setting $\psi^{*}=\psi\left(\mathbf{V}^{*}\right)$, there exist a subsequence $\left\{\psi\left(\mathbf{V}^{k^{\prime \prime}}\right)\right\}$ of $\left\{\psi\left(\mathbf{V}^{k^{\prime}}\right)\right\}$ and a $\psi^{*} \in W(0, T)$ such that

$$
\psi\left(\mathbf{V}^{k^{\prime \prime}}\right) \rightarrow \psi^{*} \text { weakly in } L^{2}(0, T ; \mathscr{H}) \cap L^{2}(0, T ; \mathscr{V}) .
$$

as $k^{\prime \prime} \rightarrow \infty$. Since the embedding $\mathscr{V} \hookrightarrow \mathscr{H}$ is compact, from the Aubin-Lions-Temam theorem (cf. [11]), then there is $\bar{\psi} \in \mathscr{H}$ that

$$
\psi\left(\mathbf{V}^{k^{\prime \prime}}\right) \rightarrow \bar{\psi} \text { strongly in } L^{2}(0, T ; \mathscr{H}) \quad k^{\prime \prime} \rightarrow \infty,
$$

and get the convergences for $\psi$ as

$$
\begin{aligned}
& \frac{\partial \psi}{\partial t}\left(\mathbf{V}^{k^{\prime \prime}}\right) \rightarrow \frac{\partial \bar{\psi}}{\partial t} \text { weakly in } L^{2}\left(0, T ; \mathscr{V}^{\prime}\right), \\
& \nabla \psi\left(\mathbf{V}^{k^{\prime \prime}}\right) \rightarrow \nabla \bar{\psi} \text { weakly in } L^{2}(0, T ; \mathscr{H}),
\end{aligned}
$$

as $k^{\prime \prime} \rightarrow \infty$. Set $\psi^{k^{\prime \prime}}=\psi\left(\mathbf{V}^{k^{\prime \prime}}\right)$, therefore, for $N$ particles $\left(P_{1}, \cdots, P_{N}\right)$, by taking test function $w^{j}=\eta$, by the definition of weak form (3) to find

$$
\int_{0}^{T}-\mathbf{i} \hbar \psi^{k^{\prime \prime}} \frac{\partial \eta}{\partial t} d \mathbf{x} d t=\int_{0}^{T} \int-\frac{\hbar^{2}}{2 M} \frac{\partial \psi^{k^{\prime \prime}}}{\partial \mathbf{x}} \frac{\partial \eta}{\partial \mathbf{x}}+\mathbf{V}^{k^{\prime \prime}} \psi^{k^{\prime \prime}} \eta+N v\left|\psi^{k^{\prime \prime}}\right|^{2} \psi^{k^{\prime \prime}} \eta d \mathbf{x} d t
$$


If using (6), (7)-(8), and taking $k^{\prime \prime} \rightarrow \infty$ to yield that

$$
\begin{aligned}
& \int_{0}^{T}-\mathbf{i} \hbar \bar{\psi} \frac{\partial \eta}{\partial t} d \mathbf{x} d t \\
= & \int_{0}^{T}-\frac{\hbar^{2}}{2 M} \frac{\partial \bar{\psi}}{\partial \mathbf{x}} \frac{\partial \eta}{\partial \mathbf{x}}+\mathbf{V}^{*} \bar{\psi} \eta+N v|\bar{\psi}|^{2} \bar{\psi} \eta d \mathbf{x} d t \quad \forall \eta \in C^{1}(0, T ; \mathscr{V}) .
\end{aligned}
$$

It inferred from (9) that $\bar{\psi}$ is a solution of (1) in the sense of distribution $\mathscr{D}^{\prime}(0, T)^{N}$ on $(0, T)$. From the uniqueness of weak solution for BEC system to attain $\bar{\psi}=\psi\left(\mathbf{V}^{*}\right)$. For $\psi$ to get two convergences as

$$
\begin{aligned}
& \psi\left(\mathbf{V}^{k^{\prime \prime}}\right) \rightarrow \psi\left(\mathbf{V}^{*}\right) \quad \text { strongly in } L^{2}(0, T ; \mathscr{H}), \\
& \psi^{f}\left(\mathbf{V}^{k^{\prime \prime}}\right) \rightarrow \psi^{f}\left(\mathbf{V}^{*}\right) \quad \text { strongly in } \mathscr{H}, \quad k^{\prime \prime} \rightarrow \infty .
\end{aligned}
$$

Since the norm $\|\cdot\|_{\mathbf{L}^{2}(\Omega)}$ are lower semi-continuous in weak topology of $\mathscr{H}$, for $N$ particles

$$
\liminf _{k^{\prime \prime} \rightarrow \infty}\left\|\psi^{f}\left(\mathbf{V}^{k^{\prime \prime}}\right)-\psi^{\text {target }}\right\|_{\mathscr{H}}^{2} \geq\left\|\psi^{f}\left(\mathbf{V}^{*}\right)-\psi^{\text {target }}\right\|_{\mathscr{H}}^{2}
$$

Vice versa, from weak convergence (6) that $\liminf _{k^{\prime \prime} \rightarrow \infty}\left(\mathbf{V}^{k^{\prime \prime}}, \mathbf{V}^{k^{\prime \prime}}\right) \mathbf{U} \geq\left(\mathbf{V}^{*}, \mathbf{V}^{*}\right) \mathbf{U}$ at $L^{2}(Q)^{N}$. To cost function (5), $J=\liminf _{k^{\prime \prime} \rightarrow \infty} J\left(\mathbf{V}^{k^{\prime \prime}}\right) \geq J\left(\mathbf{V}^{*}\right)$, and $J\left(\mathbf{V}^{*}\right)=\inf _{\mathbf{V} \in \mathbf{U}_{a d}} J(\mathbf{V})$. That is means, $\mathbf{V}^{*}(t, \mathbf{x})$ is quantum optimal control to criteria function (5). It is Theorem 4.

Theorem 5 [Optimality system] For $\psi_{0} \in \mathscr{V}$, and control problem for G-P equation (1) to cost function (5), if $\mathbf{U}_{a d}$ is closed convex (bounded) admissible subset of $\mathbf{U}$, then quantum optimal control $\mathbf{V}^{*}$ is characterized by Euler-Lagrange system as

$$
\begin{aligned}
& \left\{\begin{array}{l}
\mathbf{i} \hbar \frac{\partial \psi}{\partial t}=-\frac{\hbar^{2}}{2 M} \Delta \psi+\mathbf{V}^{*} \psi+N v|\psi|^{2} \psi \text { in } \mathrm{Q}, \\
\psi\left(\mathbf{V}^{*}, 0\right)=\psi_{0} \text { on } \Omega
\end{array}\right. \\
& \left\{\begin{array}{l}
\mathbf{i} \hbar \frac{\partial \sigma}{\partial t}=-\frac{\hbar^{2}}{2 M} \Delta \sigma+2 N v \psi \psi \sigma+N v \psi^{2} \sigma \text { in } \mathrm{Q}, \\
\mathbf{i} \sigma(T)=\psi^{f}\left(\mathbf{V}^{*}\right)-\psi^{\text {target }} \text { on } \Omega,
\end{array}\right. \\
& \int_{0}^{T} \sigma\left(\mathbf{V}^{*}\right)\left(\mathbf{V}-\mathbf{V}^{*}\right) d \mathbf{x} d t+\left(\mathbf{V}^{*}, \mathbf{V}-\mathbf{V}^{*}\right) \mathbf{U} \geq 0 \quad \forall \mathbf{V} \in \mathbf{U}_{a d} .
\end{aligned}
$$

In here, $\sigma \in W(0, T)$ is weak solution of adjoint system (11) to solution $\psi$ of BEC system (10). It is known that (12) is necessary optimality condition for quantum optimal control $\mathbf{V}^{*}(t, \mathbf{x})$ of $N$ particles $\left(P_{1}, \cdots, P_{N}\right)$.

\section{Bang-Bang Principle}

Take admissible subset $\mathscr{U}_{a d}$ of control space $\mathscr{U}$ as

$$
\begin{aligned}
\mathbf{U}_{a d}=\mathscr{U}_{a d}^{N}=\left\{V_{n} \mid V_{n}=V_{n}^{1}+V_{n}^{2}+V_{n}^{3}, V_{n}^{1} \in[0, T] \times\left[V_{n}^{1 a}, V_{n}^{1 b}\right] ;\right. \\
\left.V_{n}^{2} \in[0, T] \times\left[V_{n}^{2 a}, V_{n}^{2 b}\right] ; V_{n}^{3} \in[0, T] \times\left[V_{n}^{3 a}, V_{n}^{3 b}\right]\right\}^{N} .
\end{aligned}
$$

That is, for a.e. $t \in[0, T]$

$$
V_{n}^{1 a}(t) \leq V_{n}^{1}(t) \leq V_{n}^{1 b}(t), \quad V_{n}^{2 a}(t) \leq V_{n}^{2}(t) \leq V_{n}^{2 b}(t), \quad V_{n}^{3 a}(t) \leq V_{n}^{3}(t) \leq V_{n}^{3 b}(t)
$$


to make $V_{n}^{a}(t, \mathbf{x}) \leq V_{n}(t, \mathbf{x}) \leq V_{n}^{b}(t, \mathbf{x})$ for

$$
V_{n}^{a}=V_{n}^{1 a}+V_{n}^{2 a}+V_{n}^{3 a}, \quad V_{n}^{b}=V_{n}^{1 b}+V_{n}^{2 b}+V_{n}^{3 b} .
$$

Set $\mathbf{V}^{a}=\left(V_{1}^{a}, \cdots, V_{N}^{a}\right), \mathbf{V}^{b}=\left(V_{1}^{b}, \cdots, V_{N}^{b}\right)$, hence $\mathbf{V}^{a} \leq \mathbf{V} \leq \mathbf{V}^{b}$. Then, at the necessary optimality condition (12), consider system cost of first term at cost function (5) (taking $\varepsilon^{2}=0$ ) to get

$$
\int_{0}^{T} \sigma\left(\mathbf{V}^{*}\right)\left(\mathbf{V}-\mathbf{V}^{*}\right) d \mathbf{x} d t \geq 0, \quad \forall \mathbf{V} \in \mathbf{U}_{a d}
$$

From the Lebesgue convergent theorem to get, for a.e. $\mathbf{x} \in \Omega$ and $t \in[0, T]$, we have

$$
\sigma\left(\mathbf{V}^{*}\right)\left(\mathbf{V}-\mathbf{V}^{*}\right) \geq 0, \quad \forall \mathbf{V} \in \mathbf{U}_{a d}
$$

The details formulation for quantum optimal control can be determined by the sign of solution $\sigma\left(\mathbf{V}^{*}\right)$ of adjoint system as

$$
\begin{aligned}
& \text { if } \sigma\left(\mathbf{V}^{*}\right) \geq 0 \text { then } \mathbf{V}^{*}(t, \mathbf{x})=\mathbf{V}^{a}(t, \mathbf{x}) \text { for a.e. }(t, \mathbf{x}) \in Q ; \\
& \text { if } \sigma\left(\mathbf{V}^{*}\right) \leq 0 \text { then } \mathbf{V}^{*}(t, \mathbf{x})=\mathbf{V}^{b}(t, \mathbf{x}) \text { for a.e. }(t, \mathbf{x}) \in Q .
\end{aligned}
$$

It is well known, this is the Bang-Bang Principle for optimal control input $\mathbf{V}^{*}(t, \mathbf{x})$ subject to admissible subset $\mathbf{U}_{a d}$.

\section{Conclusions and Discussion}

At matter surface, quantum control for trapped Bose-Einstein-Condensate is considered with full proof of the existence of optimal control in complex Hilbert space (cf. [17]). Quantum control theory is applied to Gross-Pitaevskii equation in the framework of variational method. Bang-Bang Principle is deduced for quantum optimal control. It is a theoretical work of control trappping BEC. Computational approach and numerical demonstration would be further research direction. Physical experiments, surface at room temperature might be useful device to manipulate atoms.

As to the theoretic survey of controlling trapped Bose-Einstein-Condensates at matter surface, forthcoming works could be concentrate to computational, and experimental aspects at the physics and chemistry fields at 2D or 3D cases.

At laboratory control of particles, atom, ion at BEC status on the matter surface (e.g. control of ion $\mathrm{Yb}^{+}$in ${ }^{87} \mathrm{Rb}$ atoms BEC, ${ }^{23} \mathrm{Na}$ atoms BEC at $\mathrm{Si}$ surface, and so on) would also be a interesting future works.

\section{Acknowledgement}

The author sincere thanks to the past ACS Meetings and 251st ACS National Meetings \& Exposition for posters. 


\section{References}

[1] Bose SN 1924 Z. Phys. 26178

[2] Einstein A 1924 Sitzungsber. Preuss. Akad. Wiss. 26119253

[3] Gross EP 1961 II Nuovo Cimento 20(3) 454

[4] Pitaevskii L 2003 Internat. Ser. Monogr. Phys. 116 (Clarendon Press, Oxford UK)

[5] Grond J Winckel G Schmiedmayer J Hohenester U 2009 Phys. Rev. A 80053625

[6] Wang QF Belavkin VP 2012 Cybernetic and Physics 1(2) 144

[7] Wang QF 2009 International Scientific Conference on Physics and Control

[8] Wang QF 2016 251st ACS National Meeting \& Exposition Poster

[9] Rice SA 2000 Optical Control of Molecular Dynamics (New York, Wiley)

[10] Lions J 1971 Optimal Control of Systems Governed by Partial Differential Equations (Berlin-HeidelbergNew York; Springer-Verlag)

[11] Temam, R 1997 Infinite-Dimensional Dynamical Systems in Mechanics and Physics Second Edition Applied Mathematica Sciences 68 (Berlin-Heidelberg-New York; Springer-Verlag)

[12] Dautray R Lions J 1992 Mathematical Analysis and Numerical Methods for Science and Technology 5 Evolution Problems I (Berlin-Heidelberg-New York; Springer-Verlag) 